\title{
Vascular pulsatility in patients with a pulsatile- or continuous-flow ventricular assist device
}

\author{
Adam R. Travis, BE, ${ }^{a}$ Guruprasad A. Giridharan, $\mathrm{PhD},{ }^{a}$ George M. Pantalos, PhD, ${ }^{a}$ Robert D. Dowling, MD, ${ }^{a}$ \\ Sumanth D. Prabhu, MD, ${ }^{\mathrm{b}}$ Mark S. Slaughter, MD, ${ }^{\mathrm{c}}$ Mike Sobieski, RN, ${ }^{\mathrm{c}}$ Akif Undar, PhD, ${ }^{d}$ David J. Farrar, $\mathrm{PhD},{ }^{\mathrm{e}}$ \\ and Steven C. Koenig, $\mathrm{PhD}^{\mathrm{a}}$
}

From the Cardiovascular Innovation Institute $^{\mathrm{a}}$ and the Institute of Molecular Cardiology, Department of Medicine, ${ }^{\mathrm{b}}$ University of Louisville, Louisville, Ky; Advocate Christ Medical Center, ${ }^{\mathrm{c}}$ Oak Lawn, Ill; the Departments of Pediatrics, Surgery and Bioengineering, ${ }^{\mathrm{d}}$ Penn State College of Medicine, Hershey, Pa; and the Thoratec Corporation, ${ }^{\mathrm{e}}$ Pleasanton, Calif.

Funding for this project was provided by the Jewish Hospital Heart and Lung Research Foundation (Louisville, Ky) and an equipment grant from Thoratec Corporation (Pleasanton, Calif).

Robert Dowling reports consulting fees from Abiomed and Circulite. Guruprasad Giridharan reports consulting fees from Abiomed. Steven Koenig reports consulting fees from Abiomed and grant support from Thoratec. George Pantalos reports consulting fees from Abiomed and equity ownership in Transonic Systems. Mark Slaughter reports speaker fees from Thoratec. This study was reported in part by a grant from Thoratec

Received for publication July 7, 2006; revisions accepted July 7, 2006; accepted for publication Sept 25, 2006.

Address for reprints: Steven C. Koenig, $\mathrm{PhD}$, Cardiovascular Innovation Institute, University of Louisville, 500 South Floyd St, Room 118, Department of Surgery, University of Louisville, Louisville, KY 40202 (E-mail: sckoen01@gwise.louisville.edu).

J Thorac Cardiovasc Surg 2007;133:517-24

$0022-5223 / \$ 32.00$

Copyright (c) 2007 by The American Association for Thoracic Surgery

doi:10.1016/j.jtcvs.2006.09.057
Objective: We sought to investigate differences in indices of pulsatility between patients with normal ventricular function and patients with heart failure studied at the time of implantation with continuous-flow or pulsatile-flow left ventricular assist devices.

Methods: Eight patients with normal ventricular function and 22 patients with heart failure were studied. A high-fidelity aortic and left ventricular pressure catheter was inserted retrograde through the aortic valve into the left ventricle, and transit-time flow probes were placed on the aorta and device outflow graft. Hemodynamic waveforms were recorded at native heart rate before cardiopulmonary bypass and over a range of device flow rates controlled by adjusting beat rate or rpm. These data were used to calculate vascular input impedance and 2 indices of vascular pulsatility: energy-equivalent pressure and surplus hemodynamic energy.

Results: At low support levels, pulsatile support restored surplus hemodynamic energy to within $2.5 \%$ of normal values, whereas continuous support diminished surplus energy by more than $93 \%$. At high support levels, pulsatile support augmented surplus energy by $49 \%$ over normal values, whereas continuous support further diminished surplus energy by $97 \%$. Pulsatile support diminished vascular impedance from baseline failure values, whereas continuous support increased impedance. Vascular impedances at baseline for patients undergoing pulsatile and continuous support and during pulsatile support revealed normal vascular compliance, whereas impedance during continuous support indicated a loss of compliance (or "stiffening") of the vasculature.

Conclusion: These results suggest that selection of device type and flow rate can influence vascular pulsatility and input impedance, which might affect clinical outcomes.

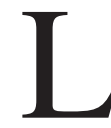
eft ventricular assist device (LVAD) implantation has become a standard therapeutic measure for bridging to transplantation in patients with end-stage heart failure and is gaining wider clinical acceptance as destination therapy in patients ineligible for transplantation, as validated by the results of the Randomized Evaluation of Mechanical Assistance for the Treatment of Congestive Heart Failure (REMATCH) study. ${ }^{1}$ Furthermore, there is hope that these devices can be operated in such a way as to promote myocardial recovery because during support, they have been shown to be capable of partially reversing many of the genetic, functional, and morphological hallmarks of the failing heart, ${ }^{2-8}$ in addition to allowing device removal without transplantation in a small fraction of patients. ${ }^{9}$ Recent evidence indicates that although there are differences in the morphology of unloading between pulsatile-flow (PF) and continuous-flow (CF) pumps, both are capable of achieving normalization of cellular damage markers, ${ }^{10}$ and although CF pumps are at an earlier stage in development, they appear to be just as effective as their older counterparts in bridging patients to transplantation. Given the 

Abbreviations and Acronyms
$\mathrm{AoF}=$ aortic flow
$\mathrm{AoP}=$ aortic pressure
$\mathrm{CF}=$ continuous flow
EEP = energy-equivalent pressure
LVAD $=$ left ventricular assist device
MAP $=$ mean arterial pressure
$\mathrm{PF} \quad=$ pulsatile flow
SHE = surplus hemodynamic energy

apparent similarity in the general hemodynamic benefit offered to patients with heart failure by the 2 device types, CF LVADs are gaining greater acceptance because they have fewer moving parts and improved mechanical reliability ${ }^{11}$ and are considerably smaller, minimizing thrombogenic surface area and enabling implantation in small adults and children.

Amidst the growing popularity of CF LVADs, however, there remain unanswered questions regarding the appropriate level of arterial pulsatility provided when using these devices. Although limited studies have shown that they might be able to provide near-physiological pulsatility at time points weeks to months after implantation, ${ }^{12}$ the degree of pulsatility achieved in human subjects with CF LVAD support has not been rigorously compared with that achieved with PF LVAD support. Diminished pulsatility with CF pumps may have significant implications for endorgan function and recovery. Investigators have shown that pulsatile arterial flow more greatly benefits the peripheral vasculature and vital organs than nonpulsatile perfusion during acute or chronic mechanical circulatory support. ${ }^{13}$ Studies of the consequences of flow character (PF vs CF) during cardiopulmonary bypass in adults ${ }^{14,15}$ have shown many important advantages of PF over nonpulsatile flow (CF or diminished PF), including decreased systemic and pulmonary vascular resistance, decreased fluid and blood requirements and less edema, decreased levels of renin and angiotensin, increased urine output and creatinine clearance, decreased thromboxane and increased prostacyclin levels, and attenuation of histologic changes associated with ischemia in brain tissue. More importantly, because LVADs are being used more frequently in destination therapy, any adverse effects of inadequate arterial pulsatility on peripheral structures might be greatly magnified with the long implantation periods associated with this type of therapy.

In this study the pulsatility achieved with CF LVADs was compared with that generated by PF LVAD support in patients with heart failure at the time of LVAD implantation. Also, the pulsatility in patients with normal ventricular function was determined to provide a normal control reference. Pulsatility was quantified by using energy-equivalent pressure (EEP) and surplus hemodynamic energy (SHE) values calculated from hemodynamic waveform measure- ments obtained intraoperatively. These metrics have been shown to provide a more physiologically relevant measure of pulsatility than the commonly reported pulse pressure. ${ }^{16-19}$ Also, the arterial load, as seen by the heart, was quantified by calculating the vascular input impedance. We hypothesized that with increasing CF LVAD support, pulsatility would diminish and afterload would increase, whereas PF LVAD support would restore pulsatility and afterload to the normal range.

\section{Patients and Methods Patients}

Two groups of patients were enrolled for this investigation: patients with normal cardiac function scheduled for coronary artery bypass grafting and patients with heart failure scheduled for LVAD implantation. The study was approved by the institutional review board at Jewish Hospital (Louisville, Ky) and Advocate Christ Medical Center (Oak Lawn, Ill), and written informed consent was obtained from all participating patients. The healthy group consisted of patients in New York Heart Association class I with normal ventricular function, which was defined as having no myocardial damage and an ejection fraction of greater than 50\%, and who were undergoing 1- to 3-vessel coronary artery bypass grafting surgery. All patients with heart failure were in New York Heart Association class IV. The type of LVAD ( $\mathrm{PF}$ or $\mathrm{CF}$ ) each patient with heart failure received was recorded, along with the cause of the cardiac disease, indication for LVAD implantation, and other pertinent descriptive data.

\section{Study Protocol}

High-fidelity hemodynamic waveforms were collected intraoperatively in the healthy patient group and in the heart failure groups at the time of PF or CF LVAD implantation. After achievement of anesthesia and immediately before initiating cardiopulmonary bypass, a transit-time flow probe (Transonics Systems, Ithaca, NY) was placed around the aortic root for measurement of aortic flow (AoF). A dualtipped, high-fidelity micromanometer catheter (Millar Instruments, Houston, Tex) was inserted into the aorta for simultaneous measurement of aortic and left ventricular pressures through a small incision made in the blood vessel (note: the same incision is used later in the procedure for inserting the infusion line for cardioplegia solution), secured with a purse-string suture, and passed retrograde through the aortic valve. Baseline hemodynamic waveforms were collected at the native heart rate and at paced heart rates $(80,100$, and 120 beats/min) in the healthy patient and heart failure groups. In the heart failure groups, after the LVAD implantation and verification of the LVAD operation (15-45 minutes), transit-time flow probes were placed around the LVAD outflow cannula and at either the aortic 
Table 1. Demographics of patients with normal ventricular function and patients with heart failure receiving continuousflow or pulsatile-flow left ventricular assist devices

\begin{tabular}{|c|c|c|c|c|c|c|}
\hline Patient no. & Sex & Age (y) & Height $(\mathrm{cm})$ & Weight (kg) & BSA & EF (\%) \\
\hline$N(n=8)$ & $5 \mathrm{M}, 3 \mathrm{~F}$ & $62 \pm 16$ & $171 \pm 10$ & $89 \pm 15$ & $2.0 \pm 0.3$ & $62 \pm 10$ \\
\hline $\mathrm{CF}(\mathrm{n}=10)$ & $7 \mathrm{M}, 3 \mathrm{~F}$ & $51 \pm 13$ & $174 \pm 10$ & $79 \pm 21$ & $1.9 \pm 0.3$ & $17 \pm 3$ \\
\hline$P F(n=12)$ & $8 \mathrm{M}, 4 \mathrm{~F}$ & $56 \pm 13$ & $176 \pm 10$ & $90 \pm 21$ & $2.1 \pm 0.3$ & $16 \pm 5$ \\
\hline
\end{tabular}

Data are expressed as means \pm standard deviations. $B S A$, Body surface area; $E F$, ejection fraction; $N$, normal ventricular function; $C F$, continuous flow; $P F$, pulsatile flow.

root or immediately downstream of the outflow cannula. The pressure catheter was also reinserted. Simultaneous hemodynamic measurements were recorded over a range of LVAD flow rates and with the LVAD temporarily turned off. LVAD flow was varied by either adjusting pump rate (PF LVAD) or rpm (CF LVAD).

\section{Data Collection and Analysis}

Hemodynamic data were signal conditioned and analog-todigitally converted at a sampling rate of $400 \mathrm{~Hz}$ in $15-$ second recording epochs and stored for digital analysis with a clinically approved Good Laboratory Practices-compliant data acquisition system. ${ }^{20,21}$ All transducers were postcalibrated against known standards to ensure measurement accuracy. Pressure and flow waveforms were used to calculate heart rate, stroke volume, cardiac output, mean aortic pressure (AoP), LVAD flow, EEP, SHE, and vascular input impedance by using a Hemodynamic Evaluation and Assessment Research Tool program ${ }^{22}$ developed in Matlab (MathWorks, Natick, Mass). Hemodynamic parameters were calculated on a beat-to-beat basis, with all beats in each data set averaged to obtain a single representative mean value. Hemodynamic parameter values for healthy and heart failure baseline values and CF and PF LVAD groups, expressed as means \pm standard error of the mean, were compared. CF and PF LVAD groups were further categorized as low or high LVAD flow support. Low LVAD flow support was defined as being the highest LVAD flow rate with native heart still ejecting, as evidenced by positive aortic root flow, positive left ventricular and AoP gradient, and opening of the aortic valve. High LVAD flow support was defined as the LVAD flow rate setting without native heart ejection through the aortic valve, as evidenced by no aortic root flow, negative left ventricular and AoP gradient, and closed aortic valve. A single-tailed $t$ test was performed to determine statistical significance.

The EEP formula is defined as the ratio of the area beneath the hemodynamic power curve $\left(\int f p d t\right)$ to the area beneath the pump flow curve $\left(\int f d t\right)$ during each pulse cycle $^{23}$ or, alternatively, the hemodynamic energy per unit volume of fluid pumped. ${ }^{16}$ It was calculated as follows: $E E P=\left(\int f p d t\right) /\left(\int f d t\right)$, where $f$ is the pump flow rate (in liters per minute), $p$ is the AoP (in millimeters of mercury), and $d t$ indicates that the integration is performed over time $(t)$. The units for the EEP are millimeters of mercury, and as such, it is possible to compare the EEP with the mean arterial pressure (MAP). The SHE value is calculated by multiplying the difference between the EEP and MAP values by the conversion factor 1332 as follows:

Table 2. Heart rate, surplus hemodynamic energy, energy-equivalent pressure, mean arterial pressure, and percentage of pulsatile energy for normal baseline, failure baseline, and low and high LVAD flow rates

\begin{tabular}{lcccccc}
\hline Condition & Device & HR (beats/min) & SHE $\left(\mathbf{e r g s} / \mathbf{c m}^{\mathbf{3}}\right)$ & EEP $(\mathbf{m m ~ H g})$ & MAP $(\mathbf{m m}$ Hg) & (EEP/MAP-1) * 100 (\%) \\
\hline Normal baseline & - & $69 \pm 5$ & $20,789 \pm 4279$ & $80.4 \pm 4.1$ & $64.8 \pm 3.3$ & 24 \\
Failure baseline & CF & $103 \pm 8$ & $17,919 \pm 1784$ & $77.6 \pm 2.8$ & $64.2 \pm 2.2$ & 21 \\
& PF & $82 \pm 4$ & $15,380 \pm 1756$ & $82.4 \pm 7.5$ & $70.8 \pm 7.4$ & 16 \\
Low VAD flow & CF & $93 \pm 13$ & $1378 \pm 471$ & $75.2 \pm 8.0$ & $74.1 \pm 7.7$ & 2 \\
& PF & $81 \pm 14$ & $20,270 \pm 4228$ & $80.8 \pm 13.0$ & $65.5 \pm 10.1$ & 23 \\
High VAD flow & CF & $103 \pm 11$ & $671 \pm 222$ & $79.8 \pm 5.3$ & $79.3 \pm 5.3$ & 6 \\
& PF & $72 \pm 10$ & $31,011 \pm 2090$ & $95.7 \pm 10.2$ & $72.4 \pm 10.8$ & 32
\end{tabular}

All results are expressed as means \pm standard error of the mean for all patients in a group. Differences between continuous-flow (CF) and pulsatile-flow (PF) left ventricular assist device (LVAD) baseline values were not statistically discernible. Differences in surplus hemodynamic energy (SHE) values between CF and PF LVADs for low $(P<.001)$ and high $(P<.000001)$ flow rates were statistically significant. Differences in SHE values between normal baseline and low PF LVAD support were not statistically discernible $(P>.4)$, suggesting the restoration of normal SHE values with low PF VAD support. Differences in SHE values between normal baseline and high PF VAD support were statistically significant $(P<.05)$, suggesting the augmentation of SHE values beyond normal baseline values. Differences in SHE values between failure baseline and low and high CF VAD support were statistically significant $(P<.001)$, suggesting the reduction of SHE values in all CF VAD support modes. $H R$, Heart rate; EEP, energy-equivalent pressure; MAP, mean arterial pressure; $V A D$, ventricular assist device. 

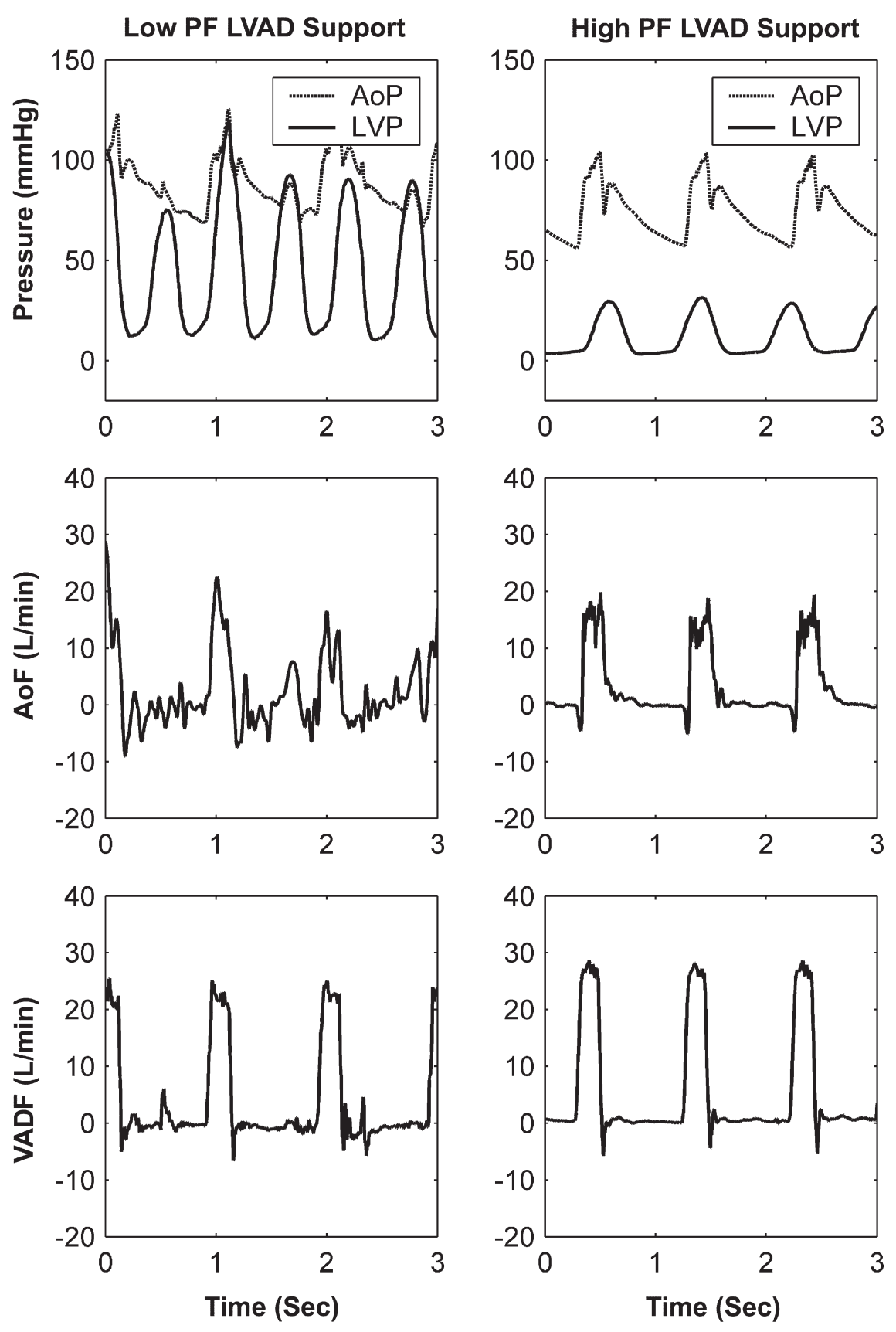

Figure 1. Aortic pressure (AoP, dotted line), left ventricular pressure (LVP, solid line), aortic distal flow (AoF), and left ventricular assist device (LVAD) flow (VADF) waveforms for low and high pulsatile-flow (PF) LVAD flow support conditions. At low PF LVAD support, the native heart still ejects through the aortic valve, as evidenced by a positive left ventricular and aortic pressure gradient and significantly increased aortic distal flow during the positive pressure gradient. At high PF LVAD flow support, the native heart does not eject through the aortic valve, as evidenced by a negative left ventricular and aortic pressure gradient.
$\operatorname{SHE}\left(\mathrm{ergs} / \mathrm{cm}^{3}\right)=1332\left[\left(\left(\int f p d t\right) /\left(\int f d t\right)\right)-M A P\right]$. This represents the extra energy required for generation of $\mathrm{PF}$ in terms of energy (not pressure) units and is thus a physiologically relevant measure of pulsatility because the generation of PF in the body is dependent on an energy gradient rather than a pressure gradient. ${ }^{23-27}$

AoP and AoF waveform recordings were used to calculate systemic vascular input impedance at baseline and over the range of LVAD flow rates. During LVAD support, the AoF was calculated as the sum of LVAD and aortic root flows. Using 15-second data epochs, the AoP and AoF waveforms were converted from time to frequency domain by using Fourier analysis algorithms in Matlab (MathWorks). The magnitudes and phases of the impedance for up to 15 harmonics were then calculated as the ratio of the magnitudes of AoP and $\mathrm{AoF}$ at each harmonic and the difference between the phases of AoP and AoF at each harmonic, respectively. An uncertainty analysis was also performed to estimate the error in the input impedance terms resulting from experimental error. 

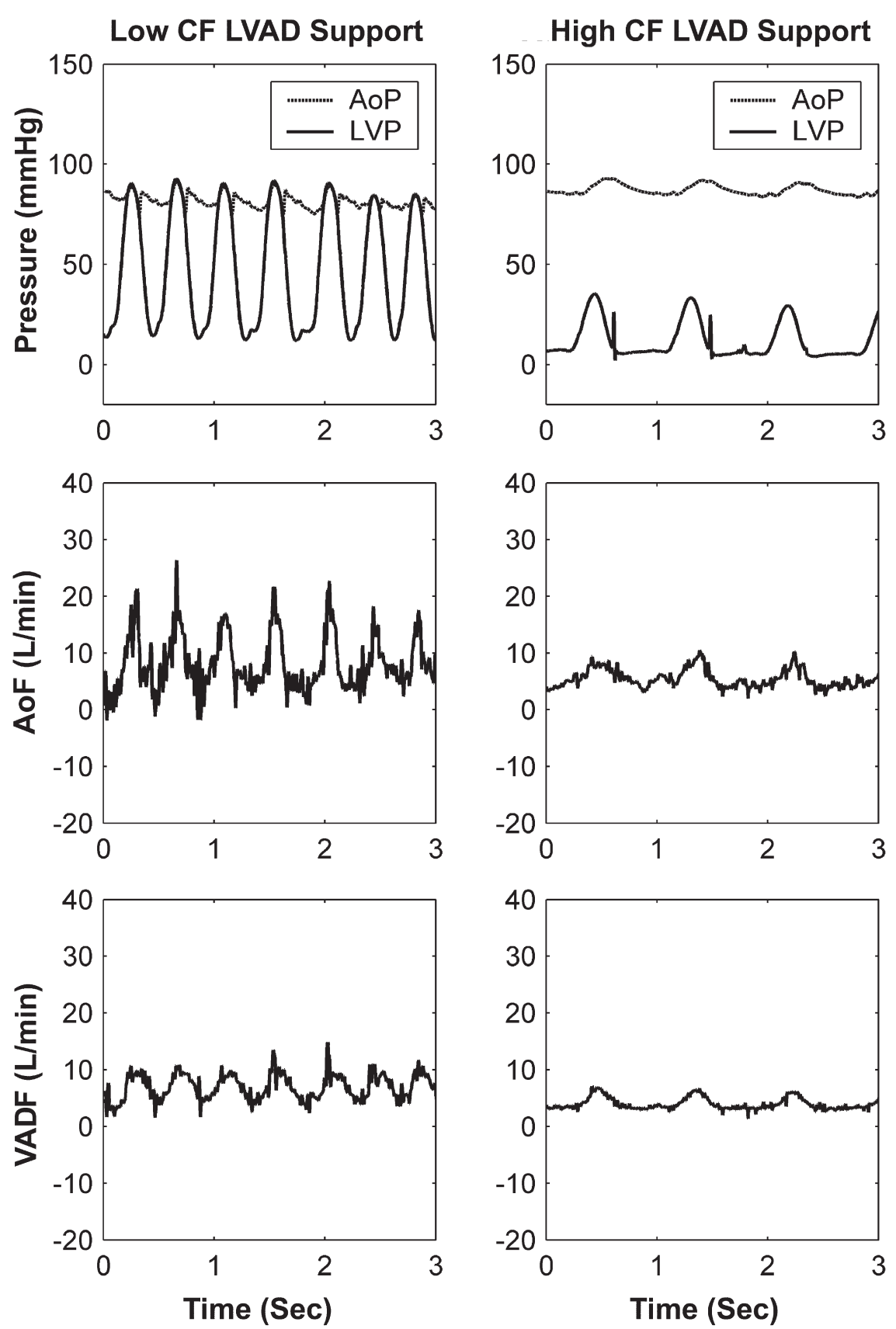
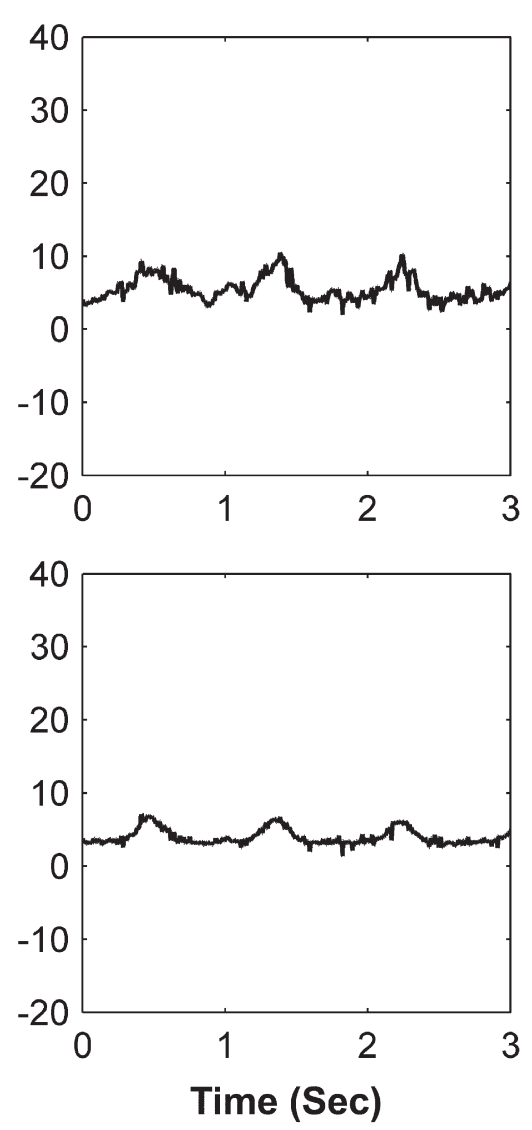

Figure 2. Aortic pressure (AoP, dotted line), left ventricular pressure (LVP, solid line), aortic distal flow (AoF), and left ventricular assist device (LVAD) flow (VADF) waveforms for low and high continuousflow (CF) LVAD flow support conditions. At Iow CF LVAD support, the native heart still ejects through the aortic valve, as evidenced by a positive left ventricular and aortic pressure gradient and significantly increased aortic distal flow during the positive pressure gradient. At high CF LVAD flow support, the native heart does not eject through the aortic valve, as evidenced by a negative left ventricular and AoP gradient.

\section{Results}

Eight healthy patients ( 5 men and 3 women) and 22 patients with heart failure (15 men and 7 women) were enrolled in this study between September 2004 and March 2006 at the Jewish Hospital Heart and Lung Institute (Louisville, Ky) and Advocate Christ Medical Center (Oak Lawn, Ill). In the group of healthy patients, the ejection fraction was $62 \% \pm 10 \%$. In the group of patients with heart failure, 11 patients had ischemic cardiomyopathy, and 11 patients had idiopathic cardiomy- opathy. The indications for implantation were 12 for bridge to transplantation, 9 for destination therapy, and 1 for bridge to recovery. Twelve patients received a PF LVAD (Thoratec HeartMate XVE, $\mathrm{n}=$ 9; Thoratec IVAD, $\mathrm{n}=3$ ), and 10 patients received a CF LVAD (Thoratec HeartMate II, $\mathrm{n}=7$; MicroMed DeBakey, $\mathrm{n}=$ 3). At the time of this writing, 1 patient had the LVAD removed on myocardial recovery, 5 had undergone transplantation, 7 had died during support, and 9 were still receiving $\mathrm{LVAD}$ support, with the average duration of 


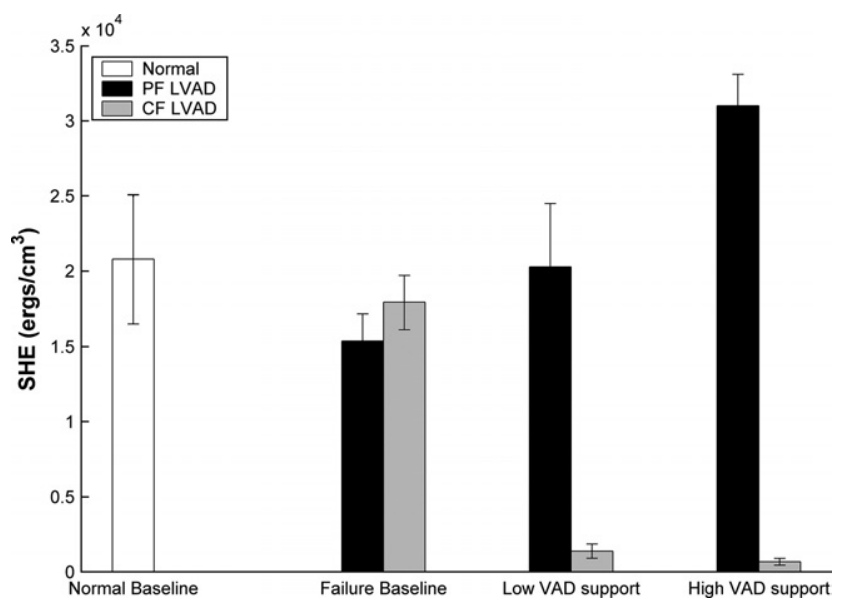

Figure 3. Comparison of surplus hemodynamic energy (SHE) between baseline normal, baseline failure, and failing left ventricles with low and high ventricular assist device (VAD) support. Although pulsatile-flow (PF) and continuous-flow (CF) left ventricular assist devices (LVAD) both restore flow, only the PF LVAD restores SHE value to normal baseline values, whereas CF LVAD decreases SHE values considerably. Differences in SHE values between CF and PF LVADs for low $(P<.001)$ and high flow rates $(P<.00001)$ were statistically significant. These data indicate a fundamental difference between levels of pulsatility achieved during CF and PF LVAD support.

support being $234 \pm 181$ days. The demographics for each patient group are summarized in Table 1 .

Patients with left ventricular failure had reduced cardiac output, EEP, and SHE values and an increased heart rate at baseline compared with patients with normal left ventricular function (Table 2). Differences in the MAP values between healthy and heart failure baseline patients were statistically insignificant (Table 2), and therefore comparisons of SHE values between groups are valid because the MAP value is fundamentally similar in all patients studied.

Support with a PF or CF LVAD restored systemic blood flow (combined aortic and LVAD blood flow) to normal baseline values. The hemodynamic waveforms suggest a diminished level of vascular pulsatility still exists with $\mathrm{CF}$ LVAD support, even at high LVAD flow rates; however, pulsatility appears much greater with PF LVAD support, as shown in Figures 1 and 2. Support with a PF LVAD augmented SHE and EEP values to normal baseline values, whereas CF LVAD support diminished SHE and EEP values (Figure 3). At low support levels, PF LVAD restored SHE values to within $2.5 \%$ of baseline normal SHE values, whereas CF LVAD diminished SHE values by more than 93\% of the normal baseline value. This effect was even more pronounced at high ventricular assist device flow rates because the PF LVAD augmented SHE values by $49 \%$ over

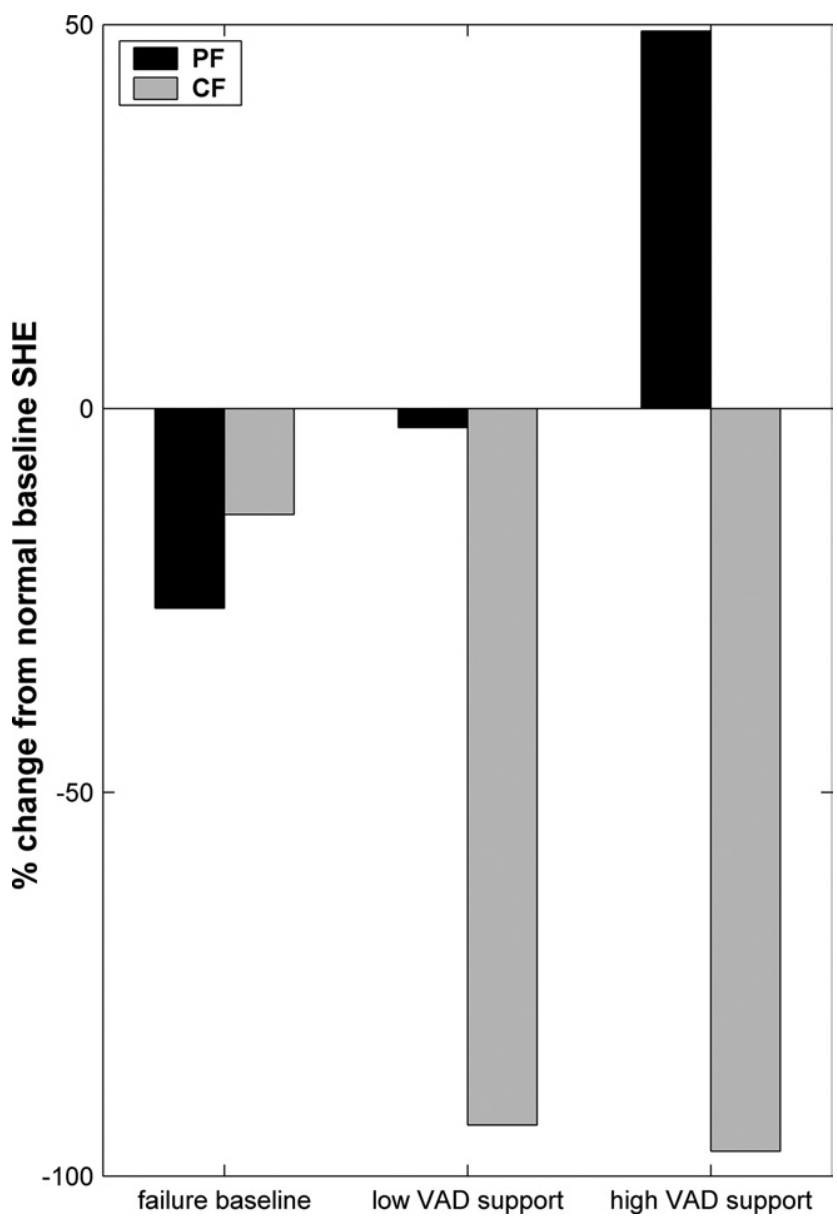

Figure 4. Comparison of percentage change in surplus hemodynamic energy (SHE) from baseline normal values for baseline failure and failing left ventricles with low and high left ventricular assist device (LVAD) support conditions. Heart failure causes a decrease in SHE values. Although pulsatile-flow (PF) and continuous-flow (CF) LVADs both restore flow, only the PF LVAD restores SHE values to normal baseline values or higher, whereas the CF LVAD decreases SHE values considerably. These data indicate a fundamental difference between levels of pulsatility achieved during CF and PF LVAD support.

baseline normal values, whereas the CF LVAD diminished SHE values by $97 \%$ (Figure 4).

The systemic vascular resistance, indicated by the impedance magnitude at $0 \mathrm{~Hz}$ (zero harmonic), decreased from failure baseline values with PF LVAD support, whereas vascular resistance increased from failure baseline values with CF LVAD support (Figure 5, A). Also, vascular impedances in patients with PF and CF LVADs at baseline and in patients with PF LVADs during support have a negative phase for the first few harmonics, which reflects normal vascular compliance, whereas impedance in patients with CF LVADs during support has a positive phase during the 

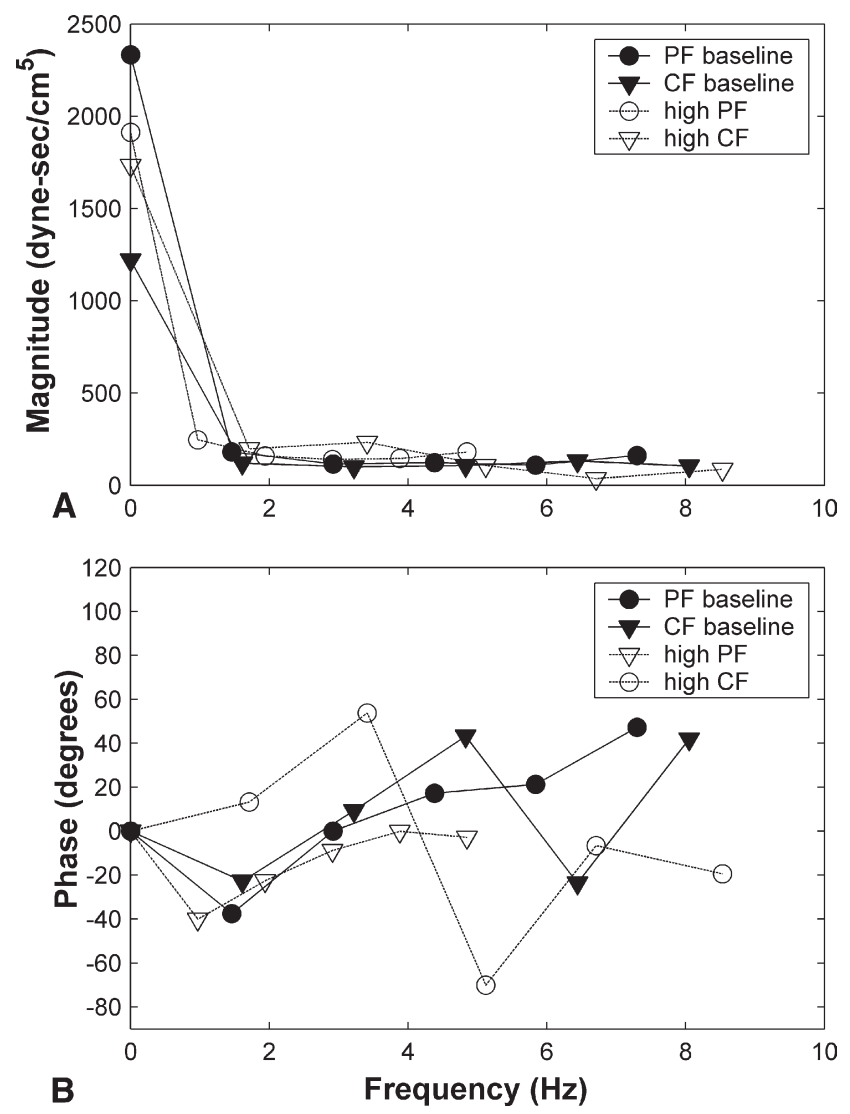

Figure 5. Comparison of vascular impedance between baseline failure and failing left ventricles supported by pulsatile-flow (PF) or continuous-flow (CF) left ventricular assist devices (LVAD) for the first 6 harmonics. A, Vascular resistance, indicated by the magnitude at $0 \mathrm{~Hz}$, decreases with PF LVAD support, whereas vascular resistance increases with CF LVAD support. B, Baseline and PF LVAD support have a negative phase for the first few harmonics, indicating normal vascular compliance. CF LVAD support has a positive phase during the first few harmonics, indicating a stiffening of the vasculature with CF LVAD support. The differences in vascular response to CF and PF LVAD support might have a significant effect on the myocardial load during LVAD weaning and might potentially affect patient outcomes.

first few harmonics, which reflects a loss of compliance (or "stiffening") of the vasculature with CF LVAD support (Figure 5, B).

\section{Discussion}

The results of this study unequivocally establish that there are substantial differences in the arterial pulsatility provided by PF and CF LVAD support at the time of device implantation. SHE values are diminished during ventricular failure compared with normal baseline values because of the loss of contractility and stroke work of the native ventricle. Furthermore, even at low levels, PF LVAD support restored
SHE and EEP values to normal baseline values. The physiologic effects of augmenting SHE and EEP values over normal baseline values, as measured during maximal PF LVAD support, are unknown. CF LVAD support caused a drastic reduction of $\mathrm{SHE}$ values, even at low levels of LVAD support, despite intermittent ejection through the aortic valve. The diminished levels of SHE and EEP with CF LVAD support are more reflective of a nonpulsatile waveform, contrary to what might be perceived visually in Figures 1 and 2. This strongly suggests that instead of allowing near-physiological pulsatility during modest support, as previously reported, ${ }^{12}$ circulatory support with a CF LVAD substantially reduces pulsatility from its physiological levels, as demonstrated by SHE values that are decreased dramatically (93\% in low support and $97 \%$ in high support) from normal baseline values. These results are in agreement with previous in vitro ${ }^{18}$ and animal ${ }^{19}$ studies, which have shown that CF LVAD support significantly diminishes vascular pulsatility.

We hypothesize that prolonged diminished pulsatility might lead to vascular stiffening that increases ventricular workload, reduces myocardial perfusion because of early (systolic) AoP wave reflections, and attenuates baroreflex sensitivity. Our results indicate that vascular impedance was reduced with PF LVAD support but was increased with $\mathrm{CF}$ LVAD support. However, our results are only indicative of acute vascular responses to LVAD support because our hemodynamic data were obtained at the time of device implantation. An extended temporal study of arterial pulsatility in chronic CF LVAD support by Potapov and colleagues ${ }^{12}$ in 2000 showed that although pulsatility was low immediately postoperatively, which is reflective of the nonpulsatile flow generated by the device and similar to our findings, pulsatility steadily increased in all patients studied over the course of CF LVAD support. The authors attributed the increase in pulsatility to increased contractility and partial recovery of the unloaded left and right ventricles. Although myocardial recovery can improve pulsatility, vascular stiffening without any improvement in myocardial function can also lead to an increase in pulsatility. Because myocardial recovery occurs only in a relatively small fraction of adults receiving LVAD support, we hypothesize that a steady chronic increase in vascular pulsatility can be attributed to vascular stiffening caused by long-term $\mathrm{CF}$ LVAD support. An extended temporal investigation of cardiovascular hemodynamic data obtained at the time of device implantation and explantation is currently underway to test this hypothesis.

\section{References}

1. Rose EA, Gelijns AC, Moskowitz AJ, et al. Long-term mechanical left ventricular assistance for end-stage heart failure. $N$ Engl $\mathrm{J} \mathrm{Med.}$ 2001;345:1435-43. 
2. Torre-Amione G, Stetson SJ, Youker KA, et al. Decreased expression of tumor necrosis factor-alpha in failing human myocardium after mechanical circulatory support: a potential mechanism for cardiac recovery. Circulation. 1999;100:1189-93.

3. Heerdt PM, Holmes JW, Cai B, et al. Chronic unloading by left ventricular assist device reverses contractile dysfunction and alters gene expression in end-stage heart failure. Circulation. 2000;102: 2713-9.

4. Dipla K, Mattiello JA, Jeevanandam V, Houser SR, Margulies KB. Myocyte recovery after mechanical circulatory support in humans with end-stage heart failure. Circulation. 1998;97:2316-22.

5. Ogletree-Hughes ML, Stull LB, Sweet WE, Smedira NG, McCarthy PM, Moravec CS. Mechanical unloading restores beta-adrenergic responsiveness and reverses receptor down-regulation in the failing human heart. Circulation. 2001;104:1241-7.

6. Levin HR, Oz MC, Chen JM, Packer M, Rose EA, Burkhoff D. Reversal of chronic ventricular dilation in patients with end-stage cardiomyopathy by prolonged mechanical unloading. Circulation. 1995;91:2717-20.

7. Zafeiridis A, Jeevanandam V, Houser SR, Margulies KB. Regression of cellular hypertrophy after left ventricular assist device support. Circulation. 1998;98:656-62.

8. Bruckner BA, Stetson SJ, Perez-Verdia A, et al. Regression of fibrosis and hypertrophy in failing myocardium following mechanical circulatory support. J Heart Lung Transplant. 2001;20:457-64.

9. Farrar DJ, Holman WR, McBride LR, et al. Long-term follow-up of Thoratec ventricular assist device bridge-to-recovery patients successfully removed from support after recovery of ventricular function. J Heart Lung Transplant. 2002;21:516-21.

10. Thohan V, Stetson SJ, Nagueh SF, et al. Cellular and hemodynamics responses of failing myocardium to continuous flow mechanical circulatory support using the DeBakey-Noon left ventricular assist device: a comparative analysis with pulsatile-type devices. J Heart Lung Transplant. 2005;24:566-75.

11. Frazier OH, Delgado RM. Mechanical circulatory support for advanced heart failure: where does it stand in 2003? Circulation. 2003; 108:3064-8.

12. Potapov EV, Loebe M, Nasseri BA, et al. Pulsatile flow in patients with a novel nonpulsatile implantable ventricular assist device. Circulation. 2000;102(suppl):III183-7.

13. Undar A. Myths and truths of pulsatile and nonpulsatile perfusion during acute and chronic cardiac support. Artif Organs. 2004;28: 439-43.

14. Nose Y. Nonpulsatile mode of blood flow required for cardiopulmonary bypass and total body perfusion. Artif Organs. 1993;17:92-102.
15. Taylor KM. Pulsatile and non-pulsatile perfusion in cardiac surgery; the continuing controversy. In: Lewis T, Graham TR, Arnold E, editors. Mechanical circulatory support.London (UK): Hodder Headline Group; 1995. p. 106-117.

16. Shepard RB, Simpson DC, Sharp JF. Energy equivalent pressure. Arch Surg. 1966;93:730-40.

17. Wright G. Hemodynamic analysis could resolve the pulsatile blood flow controversy. Ann Thorac Surg. 1994;58:1199-204.

18. Kawahito S, Nakata K, Nonaka K, et al. Analysis of the arterial blood pressure waveform using Fast Fourier Transform technique during left ventricular nonpulsatile assistance: in vitro study. Artif Organs. 2000; 24:580-3.

19. Kawahito S, Takano T, Nakata K, et al. Analysis of the arterial blood pressure waveform during left ventricular nonpulsatile assistance in animal models. Artif Organs. 2000;24:816-20.

20. Drew GA, Koenig SC. Biomedical patient monitoring, data acquisition, and playback with LabVIEWß. In: Olansen JB, Rosow E, editors. Virtual bio-instrumentation: biomedical, clinical, and healthcare applications in LabVIEW®. Upper Saddle River, NJ: Prentice Hall; 2002. p. 180-6.

21. Koenig SC, Woolard C, Drew GD, et al. Integrated data acquisition system for medical device testing and physiology research in compliance with Good Laboratory Practices. Biomed Instrum Technol. 2004; 38:229-40.

22. Schroeder MJ, Perrault B, Ewert DL, et al. HEART: an automated beat-to-beat cardiovascular analysis package using Matlab. Comput Biol Med. 2004;34:371-88.

23. Undar A, Frazier OH, Fraser CD Jr. Defining pulsatile perfusion: quantification in terms of energy equivalent pressure. Artif Organs. 1999;23:712-6.

24. Undar A, Zapanta CM, Reibson JD, et al. Precise quantification of pressure flow waveforms of a pulsatile ventricular assist device. ASAIO J. 2005;51:56-9

25. Weiss WJ, Lukic B, Undar A. Energy equivalent pressure and total hemodynamic energy associated with the pressure flow waveforms of a pediatric pulsatile ventricular assist device. ASAIO J. 2005;51:614-7.

26. Undar A, Masai T, Frazier OH, Fraser CD Jr. Pulsatile and nonpulsatile flows can be quantified in terms of energy equivalent pressure during cardiopulmonary bypass for direct comparisons. ASAIO J. 1999;45:610-4.

27. Undar A, Eichstaedt HC, Masai T, et al. Comparison of six pediatric cardiopulmonary bypass pumps during pulsatile and nonpulsatile perfusion. J Thorac Cardiovasc Surg. 2001;122:827-9. 